\title{
Análise Estatística da Distribuição de Poisson (*)
}

\author{
J. T. A. Gu $\mathbf{r} \mathbf{g}$ e 1 \\ Docente-livre \\ Seção de Genética \\ Escola Superior de Agricultura \\ "Luiz de Queiroz", Universidade \\ de S. Paulo
}

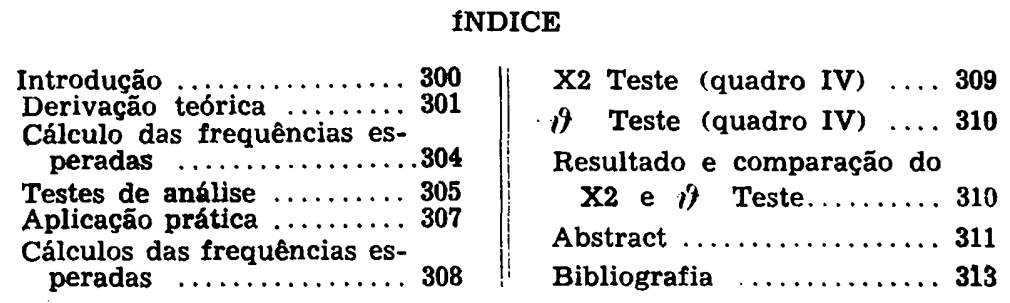

(*) Enntregue para publịcação em 2 de Agosto de 1945. 


\section{INTRODUÇAO}

A distribuição ou série de Poisson ou alnda a Lel dos pequenos números é de há muito conhecida na matemática, tendo sido publicada originalmente pelo seu autor em 1837.

$\mathrm{Na}$ estatística analítica, a série de Poisson tem sido bastante aplicada, tanto teórica como pràticamente. Assim, seguindo esta lei, encontramos na literatura exemplos sobre a distribuiçáo da frequencia em vários tipos de acidentes humanos( na indústria, no tráfego, etc.), a diluiçăo de microorganismos e posterior contagem no hematímetro, a frequência de emissáo das particulas $a$ do polónio em pequenos intervalos, a frequencia de algumas doenças em plantas e animais, etc.

Todavia, a-pesar-de ter sido já bem estudada, os livros de estatistica trazem-na sob uma forma bastante complexa, principalmente no que se refere à sua análise. Isto dificulta a sua utilizaçăo e tentando contornar esta dificuldade, apresentamos aqui com detalhes os testes de análise e a sua aplicação a exemplos práticos encontrados no nosso trabalho sobre Citrus.

Existem vários modos de definir a distribuiçăo de Poisson. Assim, por exemplo, a definiçăo dada por ARNE FISHER no seu livro "Frequency Curves" (2), pag. 95, basela-se na teoria dos semi-invariantes, enquanto que YULE, em "Introduction to the Theory of Statistics" (11), pag. 187, chega a distribuiçăo de Poisson partindo da distribuiçăo binomial; há ainda outros autores que a definem baseando-se em consideraçбes sobbre a probabilidade de tirar bolas brancas e pretas de urnas, onde o número de bolas permanece relativamente inconstante.

Todavia, podemos reunir em 3 pontos os principais caracteres da distribuiçăo de Poisson: a) trata-se de uma série descontínua, na qual as frequencias das classes só podem ser números inteiros e positivas; b) a primeira classe teórica tem sempre o valor zero, e em consequéncia disto, a pressáo do limite zero geralmente se manifesta, tornando a distribuição assimétrica; c) a frequéncia dos acontecimentos esperados é tăo pequena em relação ao número total de acontecimentos possiveis, que a frequencia observada é pràticamente independente do número total de observações.

O teste mais utilizado para determinar se as frequências observadas não diferem daquelas esperadas na série de Poisson é o $\mathrm{X}^{2}$ teste e ùltimamente BRIEGER recomenda um novo teste de $\vartheta$, o qual já foi recentemente aplicado em dois trabalhos publicados em Bragantia, sọb os títulos de "Comporta- 
mento de variedades e progênies de fumo na resistência ao vira-cabeça" (1) e "Poliembrionia em Citrus" (6).

Reunindo assim a exposiçáo sóbre o modo de calcular as frequencias esperadas e a aplicaçăo de $\mathrm{X}^{2}$ teste, 0 processo de calcular o novo teste de $y$ e os exemplos por nós encontrados em Citrus, esperamos que o presente trabalho represente algum valor aos estudiosos da estatistica.

Queremos deixar aqui consignados os nossos agradecimentos ao Prof. BRIEGER pela critica feita no desenvolvimento de todo êste trabalho.

\section{DERIVAÇAO TEORICA}

Como já foi mencionado, poderíamos derivar a distribuição de POISSON de várias maneiras, mas aqui preferimos derivá-la partindo ùnicamente da distribuição binomial.

$\mathrm{Na}$ distribuição binomial $(p+q) n$, as probabilidades $p e$ q padem tomar valores iguais ou diferentes, porém năo excessivamente diferentes, contanto que se mantenha a igualdade $p+q=1$; 0 expoente $n$ pode variar de 1 até $\infty$. Se $p=q$, a curva é simétrica e no caso do expoente $n$ crescer até tornar-se infinitamente grande, a distribuiçăo, que era descontínua, passa a ser contínua e temos, entăo, a distribuição normal, ou de GAUSS, que é uma curva "lisa".

Se $\mathrm{p} \leftrightarrows \mathrm{q}$ e o expoente $\mathrm{n}$ é baixo, a distribuição é assimétrica e essa assimetria será tanto mais acentuada quanto maior for a diferença entre as probabilidades $p$ e $q$. Se porém o expoente $n$ crescer, a assimetria vai pràticamente desaparecendo e no caso de $n$ tomar valores muito altos, poderemos ainda aceitar a distribuiçăo como aproximadamente simétrica.

Se $p<<$, consequentemente a probabilidade $q$ aproximase de 1 , e mesmo para valores de $\mathbf{n}$ pequenos ou grandes, a distribuição fica assimétrica e deșcontínua, com uma frequencia média $\mathbf{m}$ pràticamente independente do valor $n$. Este é o limite entre a distribuiçăo binomial e a distribuição de Poisson.

Para fins práticos, aceitamos que na distribuição binomial a probabilidade $\mathrm{p}$ pode chegar até 0,05 ou $5 \%$. Aqui já notamos que o produto das probabilidades p.q $=0,05.0,95=0,0475$ aproxima-se bastante de $\mathbf{p}=0,05$.

Se a probabilidade $\mathrm{p}$ cai ainda mais, chegando por exemplo até 0,01 ou $1 \%$, e o produto p.q $=0,01.0,99=0,0099$ torna-se igual a $p$ ou 0,01 e portanto independente de $q$, que fica práticamente igual a 1.

Como já frisámos no capitulo anterior, sendo na distribui- 
çăo de POISSON a frequência esperada muito pequena em reiaçăo ao número de acontecimentos, as frequênclas ficam pràticamente independentes do número total de observaçoes.

Para provarmos isto, suponhamos que numa cidade a probabilidade diária de mortes em acidentes do transito é de $0,1 \%$ ou seja 1 pessoa em 1.000 habitantes. Numa cidade de 5.000 alinas, a probabilidade seria portanto de 5 mortes, e numa outra de 6.000 habitantes seria de 6 mortes. A menor variação possivel de mortes é um individuo inteiro e só o aumento da população da ordem de um ou mais milhares de habitantes terá efeito. Uma vez que devemos comparar séries baseadas sóbre c número esperado de mortes, isto é, no nosso experimento que varia em centenas de habitantes, o número esperado ou frequencia esperada fica independente do total.

As principais fórmulas das duas distribuiçōes discutidas sह̃o:

Distribuição binomial : $\overline{\mathbf{v}}=\mathrm{pn}$

$$
\sigma= \pm \sqrt{\mathrm{pqn}}
$$

Distribuição de Poisson: $\mathrm{m}=\mathrm{f}$ esp $=\mathrm{pn} \sigma \mathrm{i}= \pm \sqrt{\mathrm{pn}}= \pm \sqrt{\mathrm{m}}$

Por essas fórmulas é evidente que para podermos desenvolver qualquer binômio, precisamos saber o valor de $\mathbf{p}$ e de $\mathbf{n}$; 0 valor de $q$ é automàticamente definido pela fórmula $p+q=1$.

Na distribuição de POISSON, porém, precisamos saber apenas o valor de $m$. Todavia, sabemos por definiçăo que $m=p n$ e não só é desnecessário saber os valores individuais de $\mathbf{p}$ e $\mathbf{n}$, como em muitos casos nem se pode determinar êsses valores.

Para explicarmos isso, podemos citar da literatura alguns exemplos:

1) No caso clássico, tantas vezes citado, de QUETELET e VON BORTKIEWICZ, (11) sôbre o número de acidentes por coice de cavalo em alguns corpos do exército prussiano, durante 20 anos (1875-94), além do valor $\mathbf{m}$, também o valor $\mathbf{n}$ é acidentalmente conhecido. $O$ valor $m$ representa o número de acidentes e o n é o número de soldados por corpo de exército. Daí pode-se calcular o valor de $\mathrm{p}=\mathrm{m}: \mathrm{n}$

2) No exemplo da resistência do fumo a "vira-cabeça", de BRIEGER e colaboradores, (1) m representa o número médio de plantas doentes por canteiro e o n o número total de plantas por canteiro.

3) De outro lado, quando determinamos o número de bactérias em um quadradinho do hematímetro, exemplo dado por STUDENT (11), podemos sempre calcular a média m de bacté- 
rias encontradas em tódas as contagens, mas o número $\mathbf{n}$ de bactérias possiveis é desconhecido; êsse número é que corresponde, no primeiro exemplo, ao número de soldados do exército que poderiam sofrer o acidente, e no segundo exemplo, ao número total de plantas por canteiro que poderiam se tornar doentes.

Até agora usámos sempre o símbolo n como constituindo o número total de casos possíveis, mas devemos ainda distinguir claramente o número de amostras investigadas em cada caso, aue nos exemplos acima citados é respectivamente o número de corpos do exército, o número de canteiros estudados e o número de quadradinhos contados no hematímetro. Para indicar o número de amostras usaremos o símbolo a.

Os exemplos que iremos dar sóbre o número de sementes em Citrus, cai no caso do 3.0 exemplo que acabamos de discutir, isto é, onde se conhece apenas o valor de $\mathbf{m}$, que é o número médio de sementes encontradas por fruto; mas o valor $\mathbf{n}$, iúmero total de sementes possiveis, quer dizer, incluindo o número de óvulos abortivos, é desconhecido. Além disso, conhecemos também o valor de a, que é o número total de frutos contados.

Terminando êste capítulo, queremos frisar que năo existe uma só distribuição de acaso, mas que o resultado do jôgo do acaso dependerá evidentemente das condições concretas nas quais cada ensaio é realizado. Assim, os velhos estatísticos jdealizaram diferentes modalidades de tirar bolas pretas e brancas de uma urna, creando desta forma condições variadas de uma tiragem sempre de acaso. De acórdo com essa probabilidade e condiçóes pre-estabelecidas, o jôgo do acaso seguirá as séries binomial ou de BERNOULLI, a de POISSON ou enfim a de LEXIS.

A curva normal ou de GAUSS-LAPLACE, como comumente é sabido, é apenas o caso extremo da distribuição binomial, onde as probabilidades favoráveis e desfavoráveis são iguais, f 0 número de tiragem de bolas sendo considerado infinito.

A comparação de uma distribuição observada com a série de POISSON e a determinação se êsses desvios encontrados podem ser atribuidos ao jỏgo de acaso, torna-se um pouco mais difícil de que no caso da comparação com uma distribuição normal, precisando, por isso, aplicar métodos especiais. Em vez de podermos usar diretamente as tábuas existentes, vai ser geralmente necessário calcular as frequências esperadas das séries ideais de POISSON, a-pesar-de que para algumas séries, 
com valores especiais de $m$ e $n$, essas frequências já estão calculadas, de acôrdo com uma citação de YULE (11), pag. 190.

\section{CALCULO DAS F'REQUENCIAS ESPERADAS}

A distribuição de POISSON, com $\mathbf{n}$ amostras e média m por amostra, tem por limite a expressão:

$$
\begin{aligned}
& n e^{-m}\left(1+m+\frac{m^{2}}{1.2}+\frac{m^{3}}{1.2 .3}+\cdots \cdot \cdots+\frac{m^{2}}{1.2 .3 \ldots a}\right)= \\
& n \cdot e^{-m}+n \cdot e^{-m} \cdot m+n \cdot e^{-m} \cdot \frac{m^{2}}{i} \cdot-e^{-m} \cdot \frac{m^{3}}{1.2 .3}+\cdots \cdots \cdots+n \cdot e^{-m} \cdot \frac{m}{1.2 .3 \ldots a}
\end{aligned}
$$

onde os têrmos seguidos indicam o valor algébrico das frequencias das classes sucessivas.

Para o cálculo dessas frequências, precisamos fazer uma transformação logarítmica, quer usando os logaritmos natuzais, quer os decimais. Assim, teremos em forma matemática as seguinteş frequências em logaritmos:

\section{Logaritmos naturais}

1.0 têrmo: $[(-m)+\log$ nat $n]$

2.0 têrmo: $[(-m)+\log$ nat $n]+\log$ nat $m$

3.0 têrmo: $[(-m)+\log$ nat $n]+2 \log$ nat $m-\log$ nat 2

4.0 têrmo: $[(-\mathrm{m})+\log$ nat $n]+\log$ nat $m-(\log$ nat $2+$ log nat 3)

a. ${ }^{\circ}$ têrmo: $[(-m)+\log$ nat $n]+a \log$ nat $m-(\log$ nat 2 $+\log$ nat $3+\ldots \ldots+\log a)$

\section{Logaritmos decimais}

1.0 têrmo $(-m \cdot \log e)+\log n$

2.0 têrmo (-m. $\log e)+\log n+\log m$

3.0 têrmo (-m. $\log e)+\log n+2 \log m-\log 2$

4.0 têrmo (-m. $\log e)+\log n+3 \log m-(\log 2+\log 3)$

a. ${ }^{\circ}$ têrmo $(-m . \log e)+\log n+a \log m-[(\log 2+\log 3+$ ..... $\log$ a)]

O cálculo dos têrmos dessa série é muito facilitado pela or- 
ganização de quadros, dos quais daremos oportunamente um exemplo. As frequencias esperadas no cálculo devem estenderse até que o antilogarítmo da última frequencia da série atinja a ordem de centésimos.

\section{TESTES DE ANALISE}

$\mathrm{X}^{2}$ teste - teste tem a vantagem de nos permitir analisar a variaçáo de cada classe e do conjunto das classes. Porém, pode acontecer, como adiante veremos, de alguns X2 parciais serem um pouco grandes, mas não ainda significantes, porém uma vez tomados em conjunto, podem dar um resultado significante. Isto dificulta o nosso julgamento e esperamos que combinando esse teste com $0 \geqslant$ teste, poderemos resolver satisfatoriamente a questa.o.

Como sabemos, o $\mathbf{X}^{2}$ teste só é seguro para as classes onde - valor numérico da frequencia esperada é igual ou maior do que 5. Acontece que sempre as frequencias em uma ou nas duas extremidades da distribuição de POİSSON são menores do que êstes limites. Neste caso, é necessário reunir as classes, somando as suas frequencias da extremidade para dentro, até que a soma das frequencias esperadas atinja ou ultrapasse 0 valor 5 .

Uma vez calculadas as frequências esperadas, fazemos para cada classe da série um $X^{2}$, definido pela fórmula (f.obs f. esp)2: f. esp. e finalmente somamos todos os $X^{2}$ parciais.

Se na comparação dos $X^{2}$ parciais, com 1 grau de liberdade, resultar um valor menor do que 3,84 ou $5 \%$ de probabilidadee, dizemos que o $\mathrm{X}^{2}$ é insignificante; se for maior do que 6,64 ou $1 \%$ de probabilidade, dizemos que é significante. Se ficar compreendido entre éstes limites de $5 \%$ e $1 \%$, dizemos que está na região de dúvida. Para o $X^{2}$ total, o grau de liberdade é ¿gual ao número de $X^{2}$ somados menos um, quando se refere a média calculada, e $\mathrm{n}$ quando se refere a variaçăo em volta de um valor ideal.

Finalmente, para cada caso temos que verificar na tabela se o valor obtido é ou năo significante.

$\vartheta$ teste - teste teste, tendo a vantagem de mostrar se a variação total da amostra observada não difere daquela esperada teòricamente, nos dá uma melhor visáo do conjunto. Todavia, acontecendo do $\vartheta$ ser significante, torna-se difícil precisar qual a classe responsável pela variação excessiva.

Para realizarmos este teste, temos que calcular a média m, o erro "standard" $\sigma$ calculado pela fórmula comum da va- 
riação descontínua com classes e o êrro ideal $\sigma$ i, que ,como acima dissemos, para a distribuição de POISSON é igual a ${ }^{\prime} \overline{\mathrm{m}}$ Embora no cálculo déstes valores tenhamos que usar os dados obtidos da série observada, todavia náo poderemos nos esquecer dos requisitos teóricos sôbre a distribuição de POISSON, como, por exemplo, que o valor da primeira classe teorica tem que ser zero.

Para melhor compreendermos êste ponto, seria bom expiicá-lo com alguns exemplos. Nos três casos da literatura cltados, o valor da primeira classe foi de fato zero: nenhum soldado morto por coice de cavalo, nenhuma planta de fumo com "vira-cabeça" e nenhuma bactéria encontrada em um quadradinho do hematímetro.

O exemplo a ser aqui discutido do número de sementes por iruto, em Citrus, também cai na mesma categoria. Contràriamente a muitas outras plantas, onde os frutos só se desenvolvem quando têm no mínimo algumas sementes, o que aliás constitui a regra, os Citrus e outras plantas frutiferas como a macieira, pereira, etc., apresentam o fenómeno da partenocarpia, isto é, os frutos podem se desenvolver sem possuirem nenhuma semente. Assim, neste caso, a primeira classe da distribuição de POISSON tem o valor zero sementes por fruto.

Mencionaremos agora alguns exemplos de uma situação bem diferente. Num trabalho publicado em Bragantia (6), MOREIRA e GURGEL estudaram a queståo da poliembrionia $\in m$ várias espécies citricas. Aqui, o material experimental é representado pelas sementes, e năo haverá sementes que não contenham no mínimo um embriăo. $O$ problema é então analisar a poliembrionia, isto é, a ocorrência de mais de um embriáo. Dêste modo, a primeira classe da distribuição de POISSON será formada pelas sementes de um só embrião, quer dizer, com zero embriőes adicionais.

Neste exemplo, poderemos indicar a média de duas formas diferentes: a média geral do número de embríos por semente, e a média do número de embriões adicionals, também por semente; para o Calamondin, essas médias foram respectivamente de 3,7 e de 2,7. No caso de aplicarmos a distribuiçáo de POISSON, sómente o último valor deve ser utilizado.

Em um outro exemplo, tirado de dados de BRIJIGER, ainda nâo publicados, sôbre a variação do número de filleiras em espigas de milho, a situação é semelhante à da poltembrionia. Nas linhagens de milho estudadas, o número minimo de futetras encontradas nas espigas foi de 8 e a variaçăo năo inclui um aumento de fileiras simples, mas sim de pares de fileiras. 
Para aplicarmas a distribuiça de Pr,ISSON, temos que raciocinar da seguinte forma: o que desejamos saber é a virriagii.) do aparecimento de pares de filelras adicionais, e a p.imejir classe será formada pelas espigas re 8 fileiras, ou com ziro par de fileiras adicionais; a segunda classe terá 10 flleiras ou wn par de flleiras adicionais, etc..

Em virtude do que expuzemos, no cálculo da médi: e do èrro "standard" da distribuiçăo de POISSON, usando is f(er. mulas da variaçăo descontinua com classes, o primeiro centru de classe Vk é zero e os demais seguem a ordem numérica. As fórmulas a serem empregadas são:

$$
\begin{aligned}
& m=f(\text { osp })=\frac{\sum\left[f(\text { abs }) \cdot V_{k}\right]}{a} \\
& C(\text { composio })=\frac{\left[\sum f\left(\text { (abi) } \bar{j} \cdot V_{k}\right]^{2}\right.}{2}
\end{aligned}
$$$$
\sigma= \pm \sqrt{\frac{\sum\left[f(\text { obs }) \cdot V_{k}^{2}\right]-C}{a-1}}
$$$$
a_{i}= \pm \sqrt{m}
$$

onde $f$ (obs) e $f$ (esp) săo as frequencia: observadas e espera. das em cada classe $\mathrm{Vk}$ da série de POISLiON e a o número $d$. ! amostras.

Finalmente, fazemos o quociente $\vartheta=\sigma$ : oi sendo 0 grau de liberdade do dividendo of $1=a-1$ e do divisor $\mathrm{nf} 2=\infty$.

Sendo quase sempre o grau de liberdade do dividenac Eie. vado, as tábus comuns do $v$ não se prestam bem e precisamo; procurar outras, mals completas. Para os nossos exemplos uli. iizámos uma tábua de BRIEGER, ainda inédita.

Se na comparaçáo do $X^{2}$ total ou do quociente 0 resultas: um valor insignificante, concluiremos que se trata de um: ss. rie de POISSON, ou que a variaçăo original, ao redor da mé.dia $\mathrm{m}$, é de acaso.

\section{APLICAÇAO PRATICA}

Problema - Fol determinado no Calamondin e nas lajaujas Natal e Coco o número de sementes por fruto e foram al.h2.. das as frequencias dadas no quadro I. Desejamos saber se es. sas frequencias por fruto variam apenas com o jogo de ai:asi, em volta do número médio calculado sobbre todos os frutos. 
Parece-nos evidente que a variação de acaso, que nós devemos aqui aceitar como variação tedricamente esperada, deverá ser a da série de POISSON. Isto porque referindo-nos aos crittérios anteriormente estabelecidos, verificamos que: 1) trata-se de uma variação descontínua, sendo presentes $6,1,2 \ldots$ sementes por fruto, números êstes sempre inteiros e positivos; 2) as frequências sempre são baixas, de modo que devemos esperar o efeito do limite zero; 3) o número total de sementes passíveis, o qual corresponde ao número total de óvulos, inclusive os abortivos, é desconhecido, porém, pode ser aceito como muito grande em relação ao número de sementes presentes por fruto.

Podemos então proceder à análise estatística, supondo tratar-se de uma distribuiçáo de POISSON.

\section{CALCULO DAS FREQUENCIAS ESPERADAS}

Laranja Natal - (Logaritmos naturais) - Para a determinação das frequênclas esperadas pela sérte de POISSON, temos que calcular primeiramente algumas constantes e depois pela lei da formação da série facilmente obteremos todos os têrmos.

As constantes necessárias são:

$\log$ nat $m=\log$ nat $3,53=1,26130$

$(\log$ nat $n)-m=\log$ nat $150-3,53=[\log$ nat $1,5+(\log$

102) $-3,53]=(0,40547+4,60517)-3,53]=1,48064$

Como já frizámos anteriormente, a organização de uma tábua facilita muito o cálculo dos térmos da série e para isto organizámos o quadro II.

Laranja Côco - (Logaritmos decimais) - O cálculo das frequências esperadas pelos logarítmos decimais năo oferece dificuldade e só temos que fazer algumas transformações logaritmicas, nas constantes a usar na tabela.

$\log \mathrm{m}=\log 3,42=0,53403$

$(\log n)-m=\log n-\log 10 e \cdot m=\log 144-(0,43429.3,42)$

$=2,5836-1,48527=0,67309$

Para facilitar o cálculo de tôdas as frequências esperadas, organizámos o quadro III. 
Calamondin - (Logaritmos naturais). - As constantes que precisamos são as seguintes:

$\log$ nat $m=\log$ nat $3,64=1,29198$

$(\log$ nat $n)-m=\log$ nat $157-3,64=1,41625$

Sendo o modo de calcular as frequencias esperadas para éste caso uma repetição do primeiro exemplo, limitar-nos-emos a dar as frequências já calculadas (quadro IV).

\section{X2 TESTE (QUADRO IV)}

Laranjas Natal e Côco - A comparação de cada $x^{2}$ parcial com $\mathrm{nf}=1$ e do total com $\mathrm{nf}=7$, nos dois casos com médias respectivamente de 3,51 e 3,42 sementes por fruto, mostra-nos que todos os valores sáo insignificantes, isto é, abaixo de $5 \%$ limite de probabilidade. Disto concluimos que a frequência de cada classe observada náo difere daquela esperada e que o conjunto também forma uma amostra da distribuição de POISSON.

Calamondin - Fazendo-se um $x^{2}$ teste entre a frequencia observada e aquela esperada da distribuição de POISSON com média de 3,64 sementes por fruto, nota-se que $0 x^{2}$ total está justamente no limite de $5 \%$ de probabilidade. Estudando-se a distribuição dos sinais, podemos constatar que êles não se distribuem ao acaso, mas que a classe do centro, que corresponde aos frutos de 2 a 5 sementes, tem uma frequencia excessiva, epquanto que as classes extremas, com 0 a 1 semente ou com 1 a 10 sementes, mostram deficiências.

Ainda mais, $0 \mathrm{x}^{2}$ teste para a classe sem e uma semente, dá um valor bem perto do limite de $1 \%$ de probabilidade. Dal podemos concluir que se trata talvez de uma distribuição de POISSON, com um certo constrangimento da variação.

Outra prova do que acabamos de dizer acima, que os sinais não se distribuem ao acaso, é a seguinte: fazendo-se uma reuniāo das classes seguidas, o que aliás é justificado, e calculando-se finalmente os $x^{2}$ (última coluna do quadro IV), deveriamos esperar, se a distribuição dos sinais fôsse ao acaso, que a redução dos $x^{2}$ parciais, que em número calu de 8 a 5 , fósse também acompanhada pela redução dos próprios valores, dando finalmente um $x^{2}$ menor ou pelo menos da mesma dimensáo que o primitivo. Porém, tal não aconteceu e $0 x^{2}$ to- 
tal obtido, depois da reunião das classes, com um valor de 13,93 e 4 graus de liberdade é bem significante, fora do limite de $1 \%$ de probabilidade.

\section{1) TESTE (QUADRO IV)}

Laranjas Natal e Côco - A comparação do êrro calculado dirıtamente das séries observadas com o da distribuiçăo de POISSON com médias respectivamente de 3,51 e 3,42 para cada caso, dá um quociente $\vartheta$ insignificante. Dal concluimos que as du: is séries em apreço são uma variação ao acaso da distribuiçắ, de POISSON.

Calamondin - A comparação do êrro calculado diretanente com 0 o i da distribuição de POISSON, com média de 3,64 demonstra que o primeiro é significantemente menor que 1) segundo. Assim, fica mais uma vez acentuada a existencia de uma redução da variação, já mencionada no capítulo anterior.

Nas laranjas Natal e Cóco, onde o $x^{2}$ teste fol insignificante, também o $\vartheta$ teste nada mostrou de anormal. Todavia, já para o Calamondin, tomando os dois testes em conjunto, podemos dizer que a anormalidade da varlação constatada é provocada pela ausência de frutas sem sementes; a falta de tais frutos talvez possa ser explicada por razzes fisiológicas.

\section{RESULTADO E COMPARAÇAO DO X2 E DO $\vartheta$ TESTE}

Comparando $0 \mathrm{x}^{2}$ teste com $0 \vartheta$ teste, devemos esperar que de um modo geral os resultados coincidam. Assim aconteceu com os dois testes na análise das laranjas Natal e Côco, onde náo houve nenhuma razăo para supor que a variaçáo do número de sementes por fruto năo fôsse de acaso, segundo a série de POISSON.

De outro lado, para o Calamondin, $0 \vartheta$ teste demonstrou que " variação evidentemente foi mais restrita do que a variação d z uma série ideal de POISSON correspondente, não se podendo, porém, dizer em detalhe, no que consiste essa restrição. Neste pronto, $0 x^{2}$ teste mostra-se mais eficiente, porque, como aliás já foi mencionado anteriormente, as frequências das classes com poucas e muitas sementes foram deftcientes, enquanto que as classes de 4 e 5 sementes foram excessivas, sendo especialmente notável e estatisticamnte significante a falta rela- 
tiva de frutos com zero e 1 semente. Deveriamos entăo tentar procurar a causa dêsses desvios da distribuição ideal de POISSON. Talvez haja, para o Calamondin, uma impossibilidade ísiológica de produzir frutos sem sementes, o que pod:ria explicar a ausência de tais frutos.

Para provar estatisticamente esta hipótese provisória, teremos que formular o problema do seguir.te modo : é necessário fisiologicamente que os frutos tenhain no minimo uma semente e o que é variável é o númer adicional de sementes por fruto. Consequentemente, a médja m para a distribuição de POISSON torna-se igual a 2,64 sementes adicionais por fruto e o êrro ideal $\sigma$ i será igual a $₫ V \overline{2,64}= \pm 1,62 \quad 0 \quad \vartheta$ entre $0 \sigma$ calculado diretamente da frequência observada com 0 $\sigma$ i é de 1,48 : 1,62 =0,91. Sendo êste quociente ") insignificante, poderemos dizer que agora encontrámos de fato uma variação de acaso, segundo a sérle de POISSON.

Poderemos ainda fazer $0 \mathrm{x}^{2}$ teste para comprovar melhor o que acima dissemos, e como poitemos ver no quadro $\mathrm{V}$, tanto os $x^{2}$ parciais como o $x^{2}$ total for.zm insignificantes, mostrando mais uma vez que o nosso raciocinto sôbre a necessidade de haver pelo menos uma semente nos srutos de Calamondin, fol verdadeiro.

Em geral, o y teste é mais fácil de caisular, não necessita de usar logaritmos e prova de um modo sumai"o se temos ou não uma distribuição de acaso, segundo a série de POISSON. Se desejarmos um teste mals exato e detalhado, temos então de recorrer ao $\mathrm{x}^{2}$ teste, e cremos que neste caso, o trabalho adicional de cálculo será largamente compensado pelos resultados.

\section{ABSTRACT}

The general properties of POISSON distributions and their relations to the binomial distribuitions are discussed. Two methods of statistical analysis are dealt with in detail :

$\mathrm{X}^{2}$-test. In order to carry out the $\mathrm{X}^{2}$-test, the mean frequency and the theoretical frequencies for all classes are calculated. Than the observed and the calculated frequencies are compared, using the well nown formula: $f$ (obs) - $f$ (esp) 2: $f($ esp). When the expected frequencies are small, one must not forget that the value of $\mathrm{X}^{2}$ may only be calculated, if the expected frequencies are biger than 5 . If smaller values should occur, the frequencies of neighboroughing classes must ge pooled. 
As a second test reintroduced by BRIEGER, consists in comparing the observed and expected error standard of the series. The observed error is calculated by the general formula:

$$
\sigma= \pm \sqrt{\frac{\sum f \cdot V k}{n-1}}
$$

where $\mathrm{n}$ represents the number of cases.

The theoretical error of a POISSON series with mean frequency $m$ is always $\pm V \bar{m}$.

These two values may be compared either by dividing the cbserved by the theoretical error and using BRIEGER's tables for $\vartheta$ or by dividing the respective variances and using SNEDECOR's tables for F. The degree of freedom for the observed error is one less the number of cases studied, and that of the theoretical error is always infinite.

In carrying out these tests, one important point must never be overlloked. The values for the first class, even if no concrete cases of the type were observed, must always be zero, an dthe value of the subsequent classes must be $1,2,3$, etc..

This is easily seen in some of the classical experiments. For instance in BORKEWITZ example of accidents in prussian armee corps, the classes are: no, one, two, etc., accidents. When counting the frequency of bacteria, these values are: no, one, two, etc., bacteria or cultures of bacteria. Ins studies of plant diseases equally the frequencies are : no, one, two, etc., plants deseased.

Howewer more complicated cases may occur. For instance, when analising the degree of polyembriony, frequently the case of "no polyembryony" corresponds to the occurrence of one embryo per each seed. Thus the classes are not: no, one, etc., embryo per seed, but they are: no additional embryo, one additional embryo, etc., per seed with at least one embryo.

Another interestin case was found by BRIEGER in genetic studies on the number os rows in maize. Here the minimum number is of course not: no rows, but: no additional beyond elght rows. The next class is not: nine rows, but: 10 rows, since the row number varies always in pairs of rows. Thus the value of successive classes are: no additional pair of rows beyond 8 , one additional pair (or 10 rows), two additional pairs (or 12 rows) etc.. 
The application of the methods is finally shown on the hand of three examples : the number of seeds per fruit in the oranges "Natal" and "Cóco" and in "Calamondin". As shown in the text and the tables, the agreement with a POISSON series is very satisfactory in the first two cases. In the third case BRIEGER's error test indicated a significant reduction of variability, and the $\mathrm{X}^{2}$ test showed that there were two many fruits with 4 or 5 seeds and too few with more or with less seeds. Howewer the fact that no fruit was found without seed, may be taken to indicate that in Calamondin fruits are not fully parthenocarpic and may develop only with one seed at the least. Thus a new analysis was carried out, on another class basis. As value for the first class the following value was accepted: no additional seed beyond the indispensable mininum number of one seed, and for the later classes the values were: one, two, etc., additional seeds. Using this new basis for all calculations, a complete agreement of the observed and expected frequencles, of the correspondig POISSON series was obtained, thus proving that our hypothesis of the impossibility of obtaining fruits without any seed was correct for Calamondin while the other two oranges were completely parthenocarpic and fruits without seeds did occur.

\section{BIBLIOGRAFIA}

1 - BRIEGER, F. G., A. RODRIGUES LIMA \& R. FORS'IER -- Comportamento de variedades e progênies de fumo na resistencia ao "vira-cabeça". Bragantia 2: 275-294.- 1942.

2 - FISHER, Arne - Frequency curves. 1.a edition - The Macmillan Company - New York - 1922.

S - FISHER, Arne - The Mathematical Theory of Probabilities - 1.a edition - The Macmillan Company - New York - 1915.

4 - FISHER, R. A. - Statistical Methods of Research Workers - 5.a edition - Oliver and Boyd - London - 1934. 
si - MOREIRA, S. e J. T. A. GURGEL - A fertilidade do pólen e sua correlação com o número de sementes, em espéciè; e formas do gênero Citrus - Bragantia 1: 669-712 1941.

(i - MOREIRA, S., e J. T. A. GURGEL - Poliembrionia em Cjtrus - Bragantia (em impressão) - 1945.

'y - RIDER, P. R. - An Introduction to Modern Statistical Methods - 1.a edition - John Wiley and Sons, Inc. London - 1939.

\$ - SNEDECOR, G. W. - Statistical Methods - Collegiate Press, Inc., Ames - Iowa - 1938.

9 - TIPPET, L. H. C. - The Methods of Statistics - 2.a edition - Williams and Norgate - London - 1939.

10 - TRELOAR, A. E. - Elements of Statistical Reasoning 1.a edition - John Wiley and Sons, Inc. - London - 1939.

11 - YULE, E. U. and M. E. KENDALL - An Introduction to the Theory of Statistics - 11.a edition - Charles Griffin and Company - London - 1937. 


\section{QUADRO I}

Número de sementes por fruto

\begin{tabular}{|c|c|c|c|}
\hline \multirow{2}{*}{$\begin{array}{l}\text { Número de } \\
\text { sementes por } \\
\text { fruto }\end{array}$} & \multicolumn{3}{|c|}{ Frequência observada } \\
\hline & Laranja Natal & Laranja Côco & Calamondin \\
\hline 0 & 4 & $\eta$ & 0 \\
\hline 1 & 16 & 15 & 8 \\
\hline 2 & 30 & 20 & 29 \\
\hline 3 & 26 & 36 & 40 \\
\hline 4 & 31 & 30 & 38 \\
\hline 5 & 24 & 20 & 26 \\
\hline 6 & 9 & 9 & 10 \\
\hline 7 & 7 & 2 & 4 \\
\hline 8 & 1 & 3 & 2 \\
\hline 9 & 2 & 2 & - \\
\hline Total $\mathbf{n}$ & 150 & 144 & 157 \\
\hline Média m & 3,51 & 3,42 & 3,64 \\
\hline
\end{tabular}




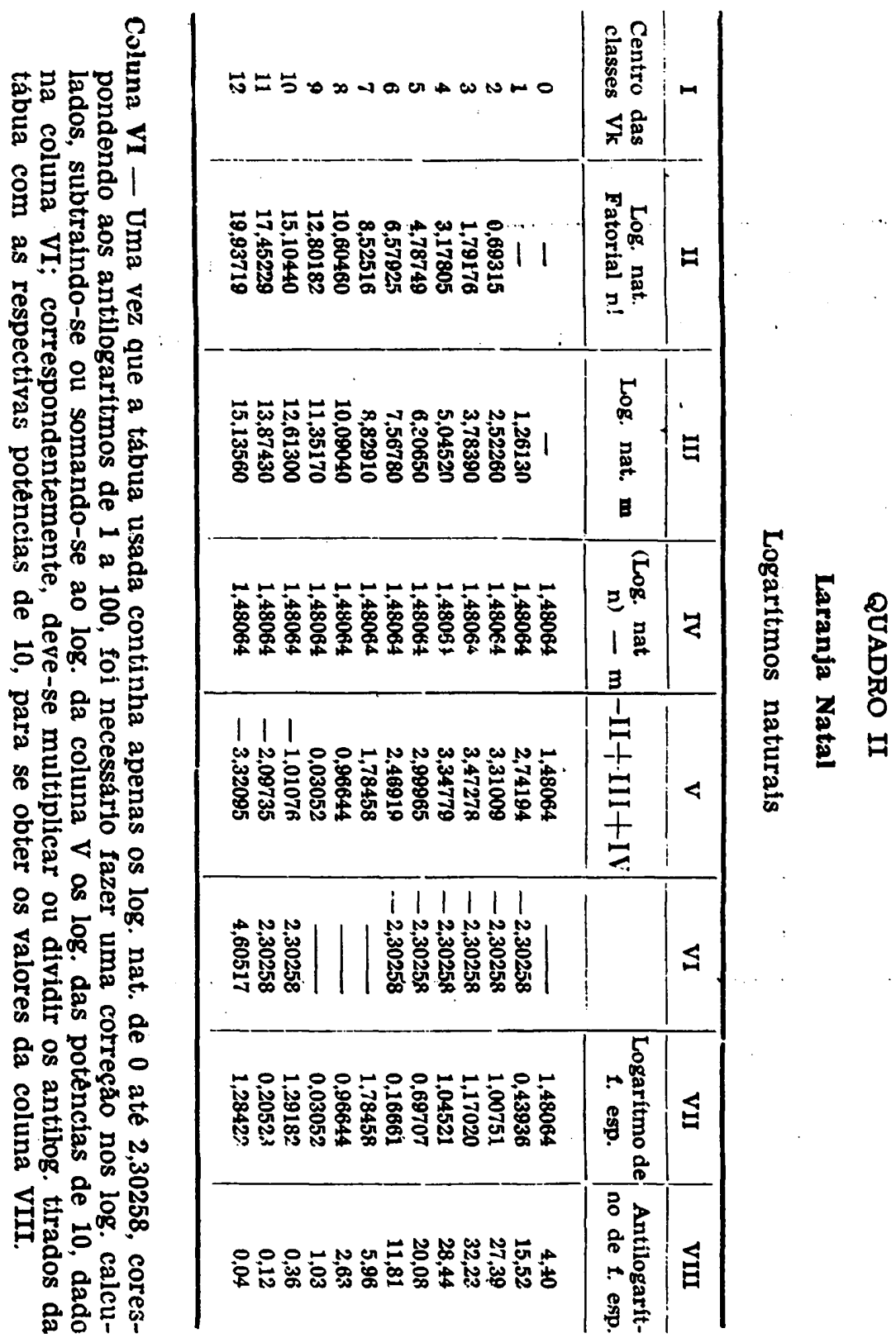




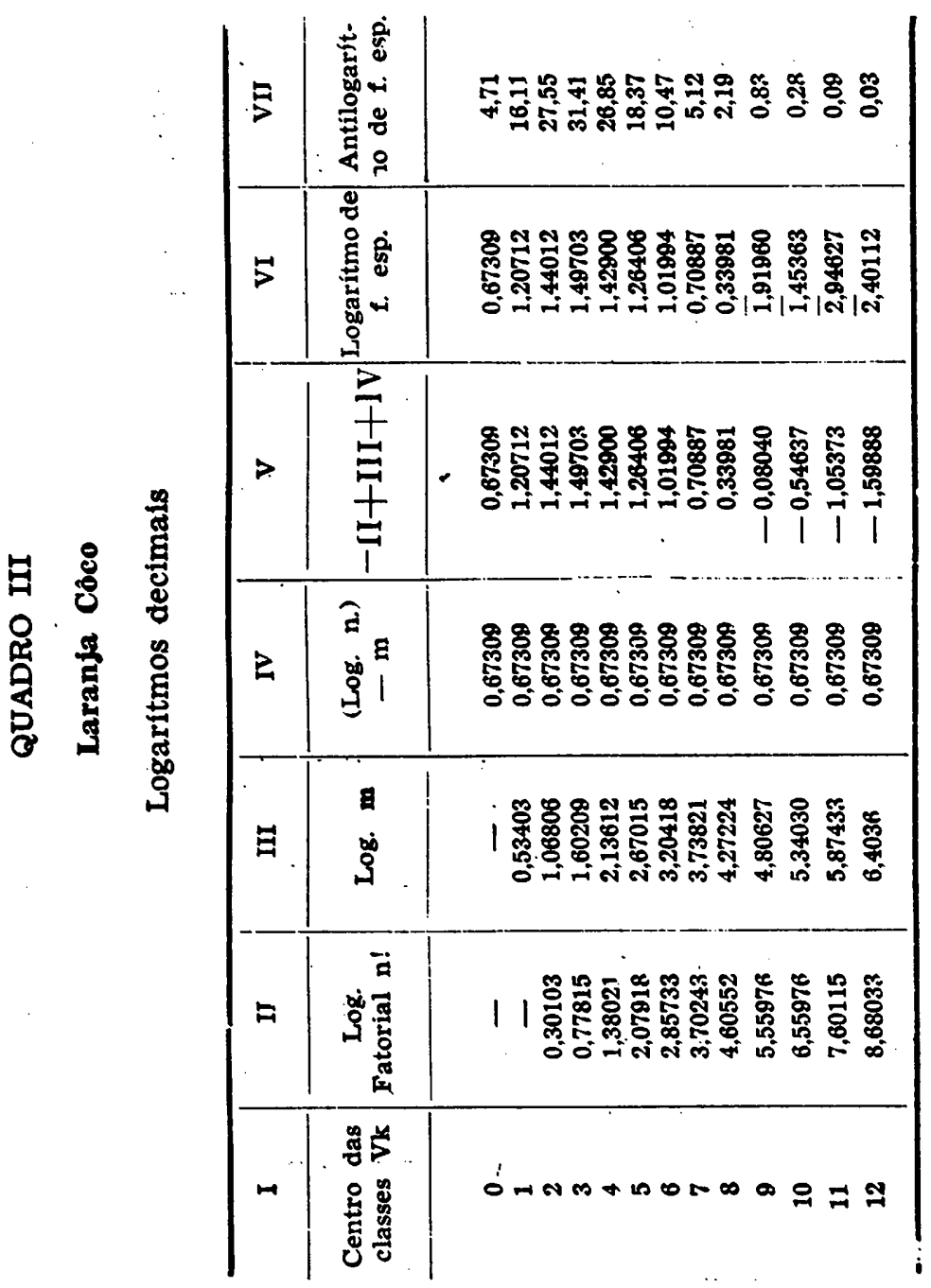




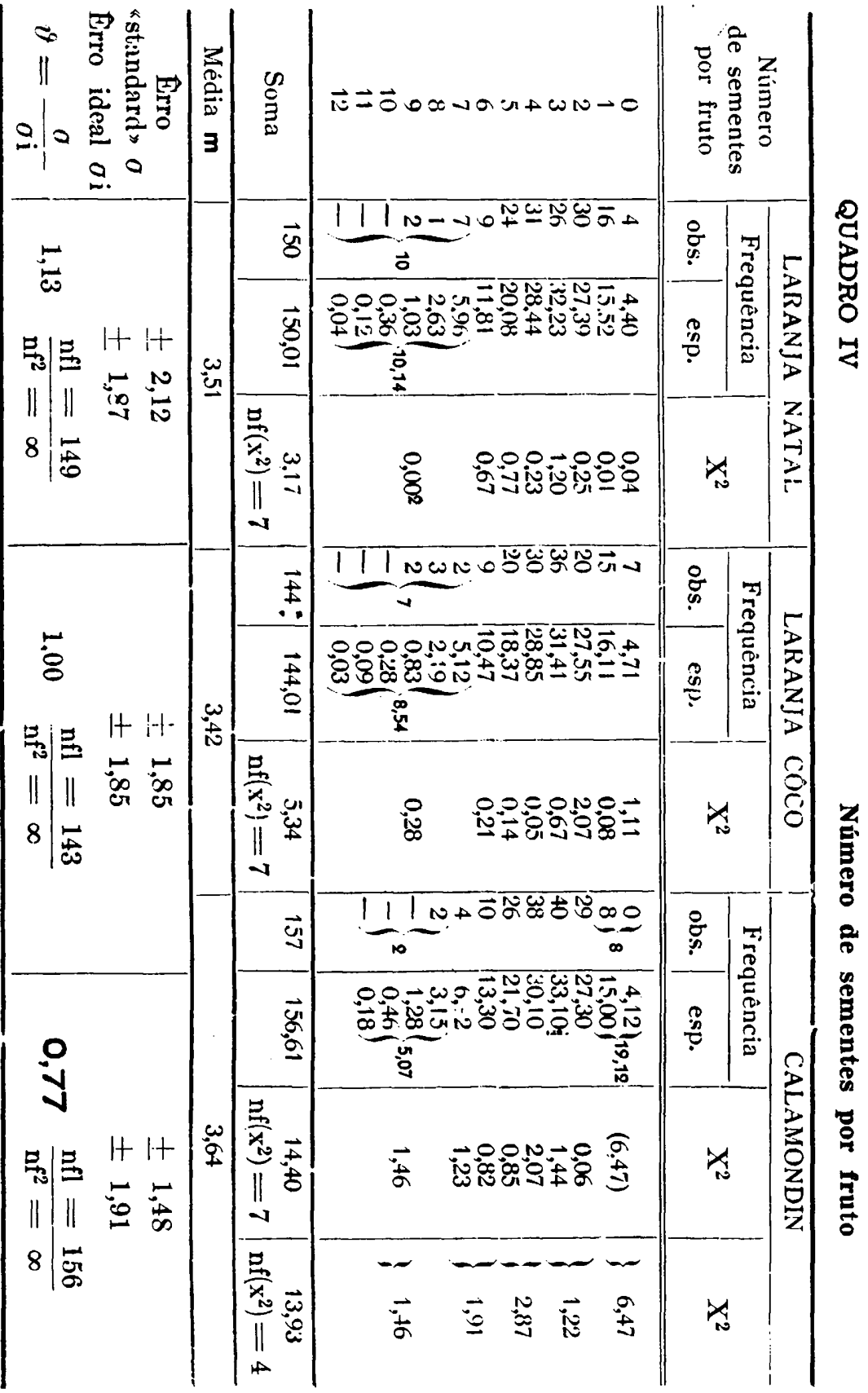




\section{QUADRO V}

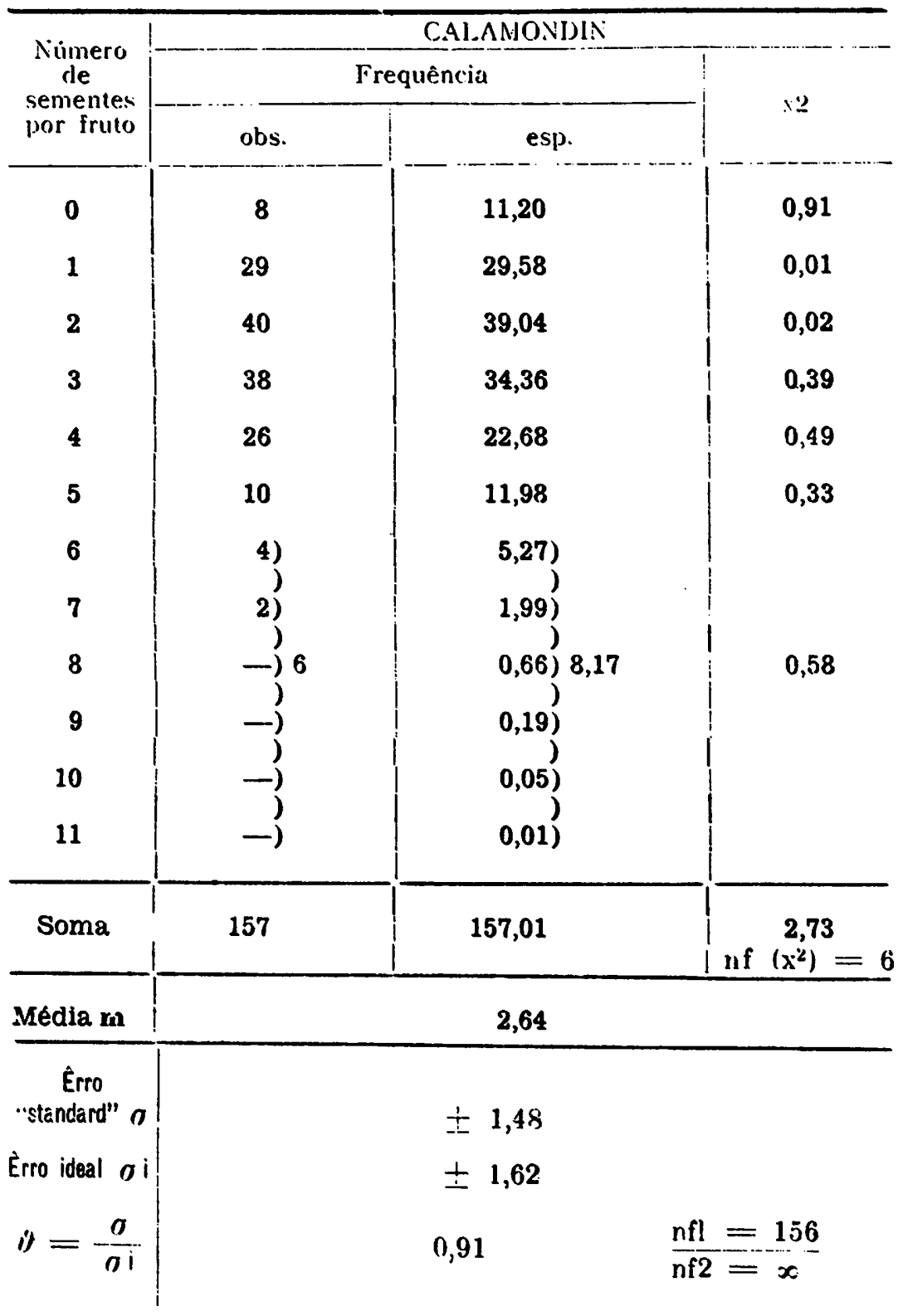

